\title{
Ammonia in positively charged pre-nucleation clusters: a quantum-chemical study and atmospheric implications
}

\author{
A. B. Nadykto, F. Yu, and J. Herb \\ Atmospheric Sciences Research Center, State University of New York at Albany, 251 Fuller Rd., Albany, NY 12203, USA
}

Received: 2 December 2008 - Published in Atmos. Chem. Phys. Discuss.: 9 February 2009

Revised: 3 June 2009 - Accepted: 5 June 2009 - Published: 18 June 2009

\begin{abstract}
The quantum-chemical treatment of prenucleation clusters consisting of atmospheric nucleation precursors is critically important for the understanding of the molecular nature of atmospheric nucleation. In the present study, the influence of ammonia on the thermochemical stability of positively charged pre-nucleation clusters has been studied using the Density Functional Theory (DFT). The formation of binary $\left(\mathrm{NH}_{4}^{+}\right)\left(\mathrm{H}_{2} \mathrm{O}\right)_{n}$ and ternary $\left(\mathrm{NH}_{4}^{+}\right)\left(\mathrm{H}_{2} \mathrm{SO}_{4}\right)\left(\mathrm{H}_{2} \mathrm{O}\right)_{n}$ ionic clusters and the conversion of $\left(\mathrm{H}_{3} \mathrm{O}^{+}\right)\left(\mathrm{H}_{2} \mathrm{O}\right)_{n-1}$ into $\left(\mathrm{NH}_{4}^{+}\right)\left(\mathrm{H}_{2} \mathrm{O}\right)_{n}$ and $\left(\mathrm{H}_{3} \mathrm{O}^{+}\right)$ $\left(\mathrm{H}_{2} \mathrm{SO}_{4}\right)\left(\mathrm{H}_{2} \mathrm{O}\right)_{n-1}$ into $\left(\mathrm{NH}_{4}^{+}\right)\left(\mathrm{H}_{2} \mathrm{SO}_{4}\right)\left(\mathrm{H}_{2} \mathrm{O}\right)_{n}$ have been investigated. The thermochemical analysis carried out in the present study shows both $\left(\mathrm{H}_{3} \mathrm{O}^{+}\right)\left(\mathrm{H}_{2} \mathrm{O}\right)_{n-1} \rightarrow\left(\mathrm{NH}_{4}^{+}\right)$ $\left(\mathrm{H}_{2} \mathrm{O}\right)_{n}$ and $\left(\mathrm{H}_{2} \mathrm{SO}_{4}\right)\left(\mathrm{H}_{3} \mathrm{O}^{+}\right)\left(\mathrm{H}_{2} \mathrm{O}\right)_{n-1} \rightarrow\left(\mathrm{NH}_{4}^{+}\right)\left(\mathrm{H}_{2} \mathrm{SO}_{4}\right)$ $\left(\mathrm{H}_{2} \mathrm{O}\right)_{n}$ transformations to be favorable thermodynamically and gives us a clear indication of the important role of ammonia in the conversion of positively charged clusters containing hydronium $\left(\mathrm{H}_{3} \mathrm{O}^{+}\right)$into those containing protonated ammonia. Under typical continental boundary layer condition, a large fraction of small positive ions may contain ammonia, but most of neutral and negative hydrated sulfuric acid monomers do not contain ammonia. In term of absolute concentrations, around $1000 \mathrm{~cm}^{-3}$ out of $10^{7} \mathrm{~cm}^{-3}$ of sulfuric acid momoners contain ammonia. $\left(\mathrm{NH}_{4}\right)^{+}\left(\mathrm{H}_{2} \mathrm{O}\right)_{n}$ clusters appear to dominate the concentrations of small positive ions. Because of the weak affinity of sulfuric acid molecules to $\left(\mathrm{H}_{3} \mathrm{O}^{+}\right)\left(\mathrm{H}_{2} \mathrm{O}\right)_{n}$ and $\left(\mathrm{NH}_{4}^{+}\right)\left(\mathrm{H}_{2} \mathrm{O}\right)_{n}$ ions $(n \leq 6)$, the concentrations of both ammoniated and un-ammoniated sulfuric acid water proton clusters are quite low. The atmospheric implications of the obtained results are discussed.
\end{abstract}

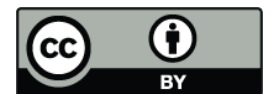

Correspondence to: $\mathrm{F}$. Yu (yfq@asrc.cestm.albany.edu)

\section{Introduction}

Atmospheric particles influence the Earth climate indirectly by affecting cloud properties and precipitation. At the present time, the aerosol indirect radiative forcing is a major source of uncertainties in understanding the climate change of the past century and predicting future climate. New particle formation is an important source of atmospheric aerosols (Kulmala et al., 2004; Yu et al., 2008). Despite the negligible contribution to the total mass of the particulate matter, secondary particles usually dominate the particle number concentration of atmospheric aerosols and cloud condensation nuclei $(\mathrm{CCN})$. The production of secondary particles is also linked to the public health because high concentrations of ultrafine (secondary) particles associated with traffic observed on and near roadways lead, according to a number of recent medical studies, to adverse health effects. A critical importance of the clear understanding and insight of the molecular nature of atmospheric nucleation for the quantitative assessment of the climate-related, health and environmental impacts of atmospheric aerosols is well established.

A strong acid at the limit of super-acidity, the sulfuric acid is the key atmospheric nucleation precursor, whose involvement in atmospheric nucleation is commonly accepted. However, the incapability of binary sulfuric acid-water homogeneous nucleation to explain the nucleation events frequently observed in the lower troposphere holds the nature of atmospheric nucleation as an unrevealed mystery. The relatively well-studied atmospheric nucleation mechanisms include: (a) binary homogeneous nucleation (BHN) of $\mathrm{H}_{2} \mathrm{SO}_{4}$ and $\mathrm{H}_{2} \mathrm{O}$ (Noppel et al., 2002; Yu., 2007); (b) ternary homogeneous nucleation (THN) of $\mathrm{H}_{2} \mathrm{SO}_{4}-\mathrm{H}_{2} \mathrm{O}-\mathrm{NH}_{3}$ (Napari et al., 2002; Yu, 2006a) ; (c) ion-mediated nucleation (IMN) of $\mathrm{H}_{2} \mathrm{SO}_{4}-\mathrm{H}_{2} \mathrm{O}$-Ion (Yu and Turco, 2000; Lovejoy et al., 2004; $\mathrm{Yu}, 2006 \mathrm{~b}$ ); and (d) organics-enhanced nucleation $\mathrm{H}_{2} \mathrm{SO}_{4}$ $\mathrm{H}_{2} \mathrm{O}$-organics (Zhang et al., 2004; Nadykto and Yu, 2007).

Published by Copernicus Publications on behalf of the European Geosciences Union. 
Although ammonia, the cornerstone of the THN, is considered as a principle stabilizer of $\mathrm{H}_{2} \mathrm{SO}_{4}-\mathrm{H}_{2} \mathrm{O}$ clusters since 1990 s, its actual role in the atmospheric nucleation remains controversial (Yu, 2006a). $\mathrm{NH}_{3}$ is well known as an efficient neutralizer of sulfuric acid solutions and in situ chemical composition measurements indicate enhanced concentrations of ammonium and sulfate in the freshly nucleated nanoparticles (6-15 nm diameter range) observed in Atlanta, Georgia (Smith et al., 2005). Nevertheless, the efficiency of $\mathrm{NH}_{3}$ as a stabilizer of small $\mathrm{H}_{2} \mathrm{SO}_{4}-\mathrm{H}_{2} \mathrm{O}$ clusters and a catalyst of the binary homogeneous nucleation is yet in question (Yu, 2006a). Classical bulk-liquid model of THN (Napari et al., 2002) faces critical difficulties in predicting the effect of ammonia on nucleation rates. While $\mathrm{NH}_{3}$ at ppt level is predicted (Napari et al., 2002) to enhance the binary nucleation by up to $\sim 30$ orders of magnitude, the enhancement in the nucleation rates measured in the laboratory studies (e.g. Ball et al., 1999; Kim et al., 1998; Christensen et al., 1992) does not exceed $\sim 2$ orders of magnitude (Yu, 2006a). The shortcomings of the work by Napari et al. (2002) have been recently corrected by Merikanto et al. (2007). Although the contribution of THN to the formation of new particles in the boundary layer is likely to be small (Yu, 2006a; Merikanto et al., 2007; Yu and Turco, 2008), the possible effect of ammonia on ion-mediated nucleation is unknown and remains to be investigated (Yu, 2006a, b). Thermochemical information about how ammonia may be involved in the pre-nucleation clusters is needed to confidently assess the role of ammonia in nucleation.

In contrast to neutral (e.g. Nadykto and Yu, 2007; Kurten et al., 2007a, b; Nadykto et al., 2008a), and, at lesser degree, negatively charged (Ortega et al., 2008, Nadykto et al., 2008b) binary and ternary clusters investigated in some detail in the past, positively charged ionic clusters containing sulfuric acid, ammonia and water are not well studied and the effect of ammonia on their formation is yet to be understood. The thermochemistry of ternary clusters/complexes is controlled by three key parameters: hydration strength and affinities of ammonia and sulfuric acid to binary clusters. The thermochemistry of $\left(\mathrm{NH}_{4}^{+}\right)$hydration has been studied by both ab initio, DFT and composite methods (Jiang, 1999; Piccard et al., 2005); however, the effect of the sulfuric acid on $\left(\mathrm{NH}_{4}^{+}\right)\left(\mathrm{H}_{2} \mathrm{O}\right)_{n}$ stability is yet to be studied.

In the present paper, the structure, properties and thermochemical stability of $\left(\mathrm{NH}_{4}^{+}\right)\left(\mathrm{H}_{2} \mathrm{SO}_{4}\right)\left(\mathrm{H}_{2} \mathrm{O}\right)_{n}$ and $\left(\mathrm{NH}_{4}^{+}\right)\left(\mathrm{H}_{2} \mathrm{O}\right)_{n}(n=1-6)$ clusters have been studied using the Density Functional Theory, focusing on the affinities of the sulfuric acid, ammonia, and water to positively charged prenucleation clusters and the formation of positively charged ternary clusters by the addition of ammonia. New thermochemical data, which can be utilized to constrain nucleation models, have been reported. The ratio of equilibrium concentrations of ternary (ammonized) and binary clusters containing different number of water molecules have been cal- culated and atmospheric implications of the obtained results have been discussed.

\section{Computational details}

The Density Functional Theory (DFT) at PW91PW91/6$311++\mathrm{G}(3 \mathrm{df} .3 \mathrm{pd})$ level has been applied to obtain the equilibrium geometries and to calculate the thermochemical properties of $\left(\mathrm{NH}_{4}^{+}\right)\left(\mathrm{H}_{2} \mathrm{SO}_{4}\right)\left(\mathrm{H}_{2} \mathrm{O}\right)_{n}$ and $\left(\mathrm{NH}_{4}^{+}\right)\left(\mathrm{H}_{2} \mathrm{O}\right)_{n}$. The previous applications of the PW91PW91 method to clusters composed of atmospheric species have shown that the aforementioned density functional is capable of providing good geometries, excellent vibrational frequencies and quite accurate free energies (Nadykto et al., 2006, 2008a, b; Kurten et al., 2006, 2007a, b, c; Ding et al., 2003; Lewandowski et al., 2005). The PW91PW91 density functional has been used in the combination with the largest Pople basis set 6-311++G(3df,3pd) providing quite small basis set superposition error (BSSE) (e.g. Muller et al., 2004; Kurten et al., 2006). Initial generated structures were first treated by semiempirical PM3 method and then by PW91PW91/6-31+G*. Finally, the most stable (within $\sim 4 \mathrm{kcal} / \mathrm{mole}$ from the lowest energy isomer) structures have been optimized at PW91PW91/6-311++G(3df,3pd) level of theory. Input structures of $\left(\mathrm{NH}_{4}^{+}\right)\left(\mathrm{H}_{2} \mathrm{O}\right)_{n}$ were build using the most equilibrium geometries obtained in Piccard et al. (2005) and Jiang (1999). Figure 1 presents the equilibrium geometries of most stable isomers of $\left(\mathrm{NH}_{4}^{+}\right)\left(\mathrm{H}_{2} \mathrm{SO}_{4}\right)\left(\mathrm{H}_{2} \mathrm{O}\right)_{n}(n=1-6)$ obtained at PW91PW91/6-311++G(3df,3pd) level of theory.

For the analysis presented in Sect. 3, the data for $\left(\mathrm{H}_{3} \mathrm{O}^{+}\right)\left(\mathrm{H}_{2} \mathrm{O}\right)_{n}$ and $\left(\mathrm{H}_{3} \mathrm{O}^{+}\right)\left(\mathrm{H}_{2} \mathrm{SO}_{4}\right)\left(\mathrm{H}_{2} \mathrm{O}\right)_{n}$ were adopted from Nadykto et al. (2006) and Nadykto et al. (2009), respectively.

\section{Results and atmospheric implications}

The explicit kinetic modeling of the evolution of atmospheric pre-nucleation clusters requires the detailed information on the thermochemistry of clusters that present in the condensable vapor mixture. The composition of the condensable vapor mixtures in the planetary atmosphere is not limited to the well-studied binary and ternary neutral clusters. Different ionic forms of unary water clusters are also present in the atmosphere. Table 1 and Fig. 2 present the comparison of the stepwise changes in hydration enthalpies and Gibbs free energies associated with the formation of $\left(\mathrm{NH}_{4}^{+}\right)\left(\mathrm{H}_{2} \mathrm{SO}_{4}\right)\left(\mathrm{H}_{2} \mathrm{O}\right)_{n}$, $\left(\mathrm{NH}_{4}^{+}\right)\left(\mathrm{H}_{2} \mathrm{O}\right)_{n},\left(\mathrm{NH}_{3}\right)\left(\mathrm{H}_{2} \mathrm{SO}_{4}\right)\left(\mathrm{H}_{2} \mathrm{O}\right)_{n},\left(\mathrm{H}_{3} \mathrm{O}^{+}\right)\left(\mathrm{H}_{2} \mathrm{O}\right)_{n}$, and $\left(\mathrm{H}_{3} \mathrm{O}^{+}\right)\left(\mathrm{H}_{2} \mathrm{SO}_{4}\right)\left(\mathrm{H}_{2} \mathrm{O}\right)_{n}$.

As seen from Table 1 and Fig. 2, the protonation of ammonia has a profound effect on the stability of hydrates containing the sulfuric acid. The hydration free energies for clusters containing protonated ammonia are $\sim 2-8 \mathrm{kcal} \mathrm{mole}^{-1}$ more 
(a)

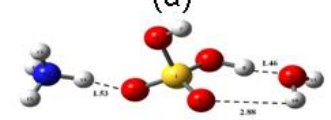

(c)

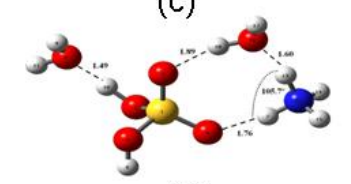

(e)
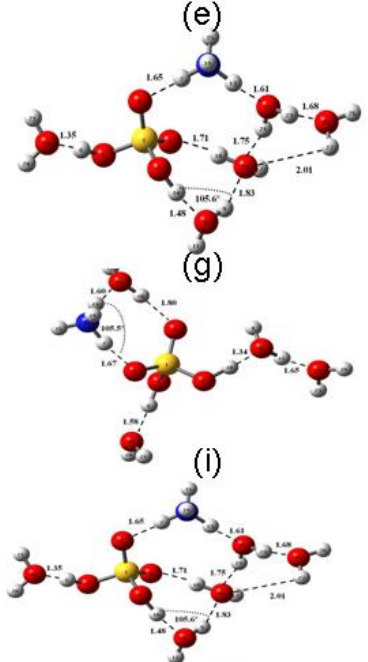

(k)

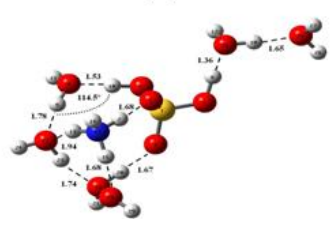

(b)

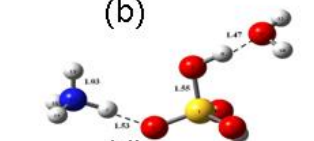

(d)

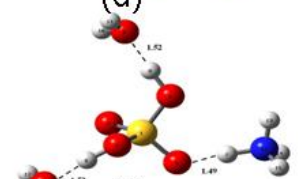

(f)

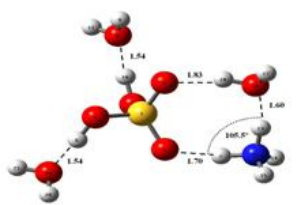

(h)

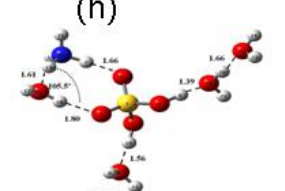

(j)

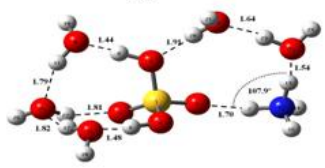

(I)

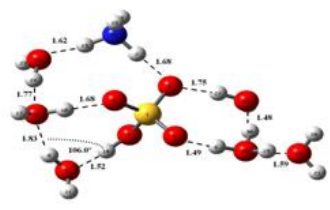

Fig. 1. Most stable isomers (two in each class) of $\left(\mathrm{NH}_{4}^{+}\right)\left(\mathrm{H}_{2} \mathrm{SO}_{4}\right)\left(\mathrm{H}_{2} \mathrm{O}\right) \quad\left(\mathbf{a} ; \quad\right.$ b $0.09 \mathrm{kcal}^{-1}$ difference in energy); $\left(\mathrm{NH}_{4}^{+}\right)\left(\mathrm{H}_{2} \mathrm{SO}_{4}\right)\left(\mathrm{H}_{2} \mathrm{O}\right)_{2}, \quad\left(\mathbf{c} ; \quad\right.$ d $\left.1.80 \mathrm{kcal} \mathrm{mole}^{-1}\right)$; $\left(\mathrm{NH}_{4}^{+}\right)\left(\mathrm{H}_{2} \mathrm{SO}_{4}\right)\left(\mathrm{H}_{2} \mathrm{O}\right)_{3}, \quad\left(\mathbf{e} ; \quad\right.$ f $\left.1.86 \mathrm{kcalmole}{ }^{-1}\right) ; \quad\left(\mathrm{NH}_{4}^{+}\right)$ $\left(\mathrm{H}_{2} \mathrm{SO}_{4}\right)\left(\mathrm{H}_{2} \mathrm{O}\right)_{4}, \quad\left(\mathbf{g} ; \quad\right.$ h $\left.0.67 \mathrm{kcal} \mathrm{mole}{ }^{-1}\right) ; \quad\left(\mathrm{NH}_{4}^{+}\right)\left(\mathrm{H}_{2} \mathrm{SO}_{4}\right)$ $\left(\mathrm{H}_{2} \mathrm{O}\right)_{5}$, (i; j $\left.0.91 \mathrm{kcal} \mathrm{mole}^{-1}\right) ;\left(\mathrm{NH}_{4}^{+}\right)\left(\mathrm{H}_{2} \mathrm{SO}_{4}\right)\left(\mathrm{H}_{2} \mathrm{O}\right)_{6}$, (k; l $\left.0.78 \mathrm{kcal} \mathrm{mole}^{-1}\right)$. Cartesian coordinates of the isomers are given in the Supplementary Information (see http://www.atmos-chem-phys. net/9/4031/2009/acp-9-4031-2009-supplement.pdf).

negative than those for $\left(\mathrm{NH}_{3}\right)\left(\mathrm{H}_{2} \mathrm{SO}_{4}\right)\left(\mathrm{H}_{2} \mathrm{O}\right)_{n}$. The comparison of the hydration free energies for $\left(\mathrm{NH}_{4}^{+}\right)\left(\mathrm{H}_{2} \mathrm{SO}_{4}\right)\left(\mathrm{H}_{2} \mathrm{O}\right)_{n}$ and $\left(\mathrm{H}_{3} \mathrm{O}^{+}\right)\left(\mathrm{H}_{2} \mathrm{SO}_{4}\right)\left(\mathrm{H}_{2} \mathrm{O}\right)_{n}$ shows that binary hydrate complexes formed over $\left(\mathrm{H}_{3} \mathrm{O}^{+}\right)$are bonded stronger than those formed over $\left(\mathrm{NH}_{4}^{+}\right)$. As may be seen from the comparison of the hydration free energies for $\left(\mathrm{NH}_{4}^{+}\right)\left(\mathrm{H}_{2} \mathrm{SO}_{4}\right)\left(\mathrm{H}_{2} \mathrm{O}\right)_{n}$ containing the sulfuric acid with those for $\left(\mathrm{NH}_{4}^{+}\right)\left(\mathrm{H}_{2} \mathrm{O}\right)_{n}$, the presence of the sulfuric acid does not enhance the hydration strength for $n<5$. The same pattern is observed in the case of $\left(\mathrm{H}_{3} \mathrm{O}^{+}\right)\left(\mathrm{H}_{2} \mathrm{SO}_{4}\right)\left(\mathrm{H}_{2} \mathrm{O}\right)_{n}$ and $\left(\mathrm{H}_{3} \mathrm{O}^{+}\right)\left(\mathrm{H}_{2} \mathrm{O}\right)_{n}$. As a matter of fact, the hydration of $\left(\mathrm{NH}_{4}^{+}\right)\left(\mathrm{H}_{2} \mathrm{O}\right)_{n}$ and $\left(\mathrm{H}_{3} \mathrm{O}^{+}\right)\left(\mathrm{H}_{2} \mathrm{O}\right)_{n}$ is

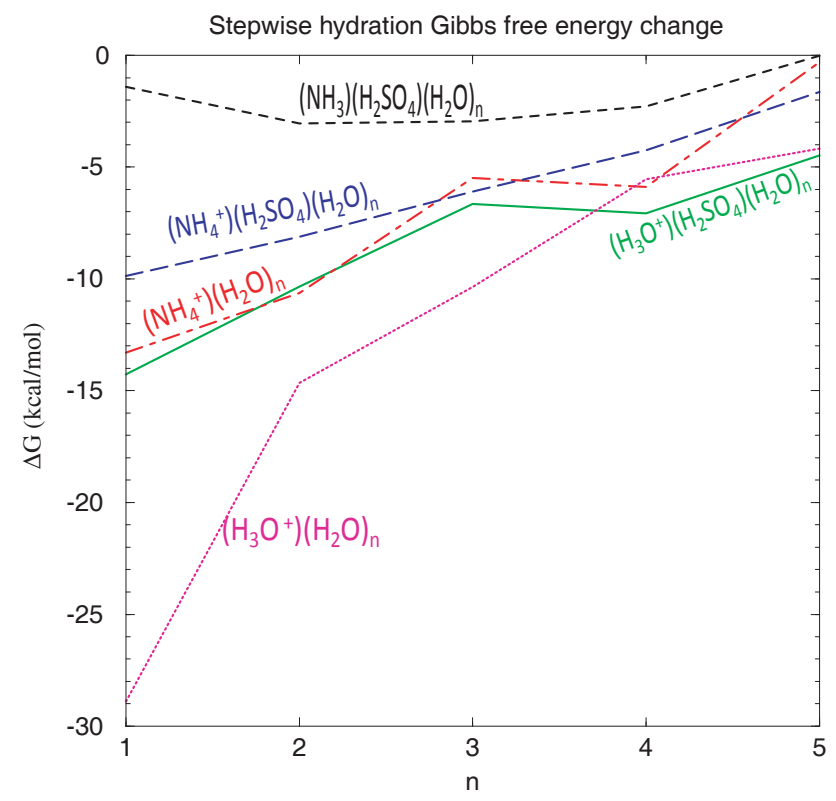

Fig. 2. Stepwise Gibbs free energy changes associated with hydration formation of $\left(\mathrm{NH}_{4}^{+}\right)\left(\mathrm{H}_{2} \mathrm{SO}_{4}\right)\left(\mathrm{H}_{2} \mathrm{O}\right)_{n},\left(\mathrm{H}_{3} \mathrm{O}^{+}\right)\left(\mathrm{H}_{2} \mathrm{SO}_{4}\right)$ $\left(\mathrm{H}_{2} \mathrm{O}\right)_{n}, \quad\left(\mathrm{NH}_{4}^{+}\right)\left(\mathrm{H}_{2} \mathrm{O}\right)_{n}, \quad\left(\mathrm{H}_{3} \mathrm{O}^{+}\right)\left(\mathrm{H}_{2} \mathrm{O}\right)_{n}$ and $\left(\mathrm{NH}_{3}\right)\left(\mathrm{H}_{2} \mathrm{SO}_{4}\right)$ $\left(\mathrm{H}_{2} \mathrm{O}\right)_{n}$.

2-4 kcal mole ${ }^{-1}$ and $1.5-14.5 \mathrm{kcal} \mathrm{mole}^{-1}$ stronger than that of $\left(\mathrm{NH}_{4}^{+}\right)\left(\mathrm{H}_{2} \mathrm{SO}_{4}\right)\left(\mathrm{H}_{2} \mathrm{O}\right)_{n}$ and $\left(\mathrm{H}_{3} \mathrm{O}^{+}\right)\left(\mathrm{H}_{2} \mathrm{SO}_{4}\right)\left(\mathrm{H}_{2} \mathrm{O}\right)_{n}$ for $n<\sim 4$. It is important to note that the difference in the hydration free energies for clusters formed over different core ions decreases quickly with the hydration number, reaching $\sim-4.5 \mathrm{kcal} \mathrm{mole}^{-1}$ at $n=5$.

The thermochemistry of the sulfuric acid, the key atmospheric nucleation precursor, is critically important for the assessment of the efficiency of ions as nucleation agents. Table 2 shows the comparison of the enthalpies and Gibbs free energies associated with the formation of $\left(\mathrm{NH}_{4}^{+}\right)\left(\mathrm{H}_{2} \mathrm{SO}_{4}\right)\left(\mathrm{H}_{2} \mathrm{O}\right)_{n}$ and $\left(\mathrm{H}_{3} \mathrm{O}^{+}\right)\left(\mathrm{H}_{2} \mathrm{SO}_{4}\right)\left(\mathrm{H}_{2} \mathrm{O}\right)_{n}$ by the addition of the sulfuric acid. As seen from Table 2, the affinities of the sulfuric acid to $\left(\mathrm{NH}_{4}^{+}\right)\left(\mathrm{H}_{2} \mathrm{O}\right)_{n}$ and $\left(\mathrm{H}_{3} \mathrm{O}^{+}\right)\left(\mathrm{H}_{2} \mathrm{O}\right)_{n}$ exhibit similar behavior. The difference in the Gibbs free energies associated with the $\left(\mathrm{NH}_{4}^{+}\right)\left(\mathrm{H}_{2} \mathrm{SO}_{4}\right)\left(\mathrm{H}_{2} \mathrm{O}\right)_{n}$ and $\left(\mathrm{H}_{3} \mathrm{O}^{+}\right)\left(\mathrm{H}_{2} \mathrm{SO}_{4}\right)\left(\mathrm{H}_{2} \mathrm{O}\right)_{n}$ formation is less than $4 \mathrm{kcal} \mathrm{mole}^{-1}$ for monohydrates and it drops to $<0.5 \mathrm{kcal} \mathrm{mole}^{-1}$ at $n=5$. On average the affinity of the sulfuric acid to protonated ammonia clusters does not exceed that of sulfuric acid to binary sulfuric acid-water clusters. It should be noted that our calculated affinity of sulfuric acid to $\left(\mathrm{H}_{3} \mathrm{O}^{+}\right)\left(\mathrm{H}_{2} \mathrm{O}\right)_{n}$ for $n>2$ is $\sim 2-4 \mathrm{kcal} / \mathrm{mol}$ less negative than the corresponding values given in Froyd and Lovejoy (2003). The reason behind the difference remains to be studied.

Table 3 presents the enthalpy and Gibbs free energy changes associated with $\left(\mathrm{H}_{3} \mathrm{O}\right)^{+}\left(\mathrm{H}_{2} \mathrm{SO}_{4}\right)\left(\mathrm{H}_{2} \mathrm{O}\right)_{n-1}$ $+\mathrm{NH}_{3} \leftrightarrow\left(\mathrm{NH}_{4}\right)^{+}\left(\mathrm{H}_{2} \mathrm{SO}_{4}\right)\left(\mathrm{H}_{2} \mathrm{O}\right)_{n},\left(\mathrm{H}_{2} \mathrm{SO}_{4}\right)\left(\mathrm{H}_{2} \mathrm{O}\right)_{n}+\mathrm{NH}_{3} \leftrightarrow$ 
Table 1. Comparison of stepwise changes in enthalpies and Gibbs free energies (kcalmole ${ }^{-1}$ ) under standard condition $(T=298.15 \mathrm{~K}$ and $P=101.3 \mathrm{KPa})$ associated with the hydration of $\left(\mathrm{NH}_{4}^{+}\right)\left(\mathrm{H}_{2} \mathrm{SO}_{4}\right)\left(\mathrm{H}_{2} \mathrm{O}\right)_{n}$ (present study), $\left(\mathrm{NH}_{4}^{+}\right)\left(\mathrm{H}_{2} \mathrm{O}\right)_{n}($ present study), $\left(\mathrm{NH}_{3}\right)\left(\mathrm{H}_{2} \mathrm{SO}_{4}\right)\left(\mathrm{H}_{2} \mathrm{O}\right)_{n}$ (from Nadykto et al., 2008a), $\left(\mathrm{H}_{3} \mathrm{O}^{+}\right)\left(\mathrm{H}_{2} \mathrm{SO}_{4}\right)\left(\mathrm{H}_{2} \mathrm{O}\right)_{n}$ (from Nadykto et al., 2009), and $\left(\mathrm{H}_{3} \mathrm{O}^{+}\right)\left(\mathrm{H}_{2} \mathrm{O}\right)_{n}($ from Nadykto et al., 2006). Data by Piccard et al. (2005) are given in parentheses.

\begin{tabular}{|c|c|c|}
\hline & $\mathrm{g} \Delta \mathrm{H}$ & \\
\hline$\left(\mathrm{NH}_{4}^{+}\right)\left(\mathrm{H}_{2} \mathrm{SO}_{4}\right)\left(\mathrm{H}_{2} \mathrm{O}\right)_{0}+\mathrm{H}_{2} \mathrm{O} \Leftrightarrow\left(\mathrm{NH}_{4}^{+}\right)\left(\mathrm{H}_{2} \mathrm{SO}_{4}\right)\left(\mathrm{H}_{2} \mathrm{O}\right)_{1}$ & -17.91 & -9.87 \\
\hline$\left(\mathrm{NH}_{4}^{+}\right)\left(\mathrm{H}_{2} \mathrm{O}\right)_{0}+\mathrm{H}_{2} \mathrm{O} \Leftrightarrow\left(\mathrm{NH}_{4}^{+}\right)\left(\mathrm{H}_{2} \mathrm{O}\right)_{1}$ & $-21.84(-20.28)$ & $-13.3(-14.11)$ \\
\hline$\left(\mathrm{H}_{3} \mathrm{O}^{+}\right)\left(\mathrm{H}_{2} \mathrm{SO}_{4}\right)\left(\mathrm{H}_{2} \mathrm{O}\right)_{0}+\mathrm{H}_{2} \mathrm{O} \Leftrightarrow\left(\mathrm{H}_{3} \mathrm{O}^{+}\right)\left(\mathrm{H}_{2} \mathrm{SO}_{4}\right)\left(\mathrm{H}_{2} \mathrm{O}\right)_{1}$ & -24.45 & -14.27 \\
\hline$\left(\mathrm{H}_{3} \mathrm{O}^{+}\right)\left(\mathrm{H}_{2} \mathrm{O}\right)_{0}+\mathrm{H}_{2} \mathrm{O} \Leftrightarrow\left(\mathrm{H}_{3} \mathrm{O}^{+}\right)\left(\mathrm{H}_{2} \mathrm{O}\right)_{1}$ & -37.84 & -28.89 \\
\hline$\left(\mathrm{NH}_{3}\right)\left(\mathrm{H}_{2} \mathrm{SO}_{4}\right)\left(\mathrm{H}_{2} \mathrm{O}\right)_{0}+\mathrm{H}_{2} \mathrm{O} \Leftrightarrow\left(\mathrm{NH}_{3}\right)\left(\mathrm{H}_{2} \mathrm{SO}_{4}\right)\left(\mathrm{H}_{2} \mathrm{O}\right)_{1}$ & -10.96 & -1.41 \\
\hline$\left(\mathrm{NH}_{4}^{+}\right)\left(\mathrm{H}_{2} \mathrm{SO}_{4}\right)\left(\mathrm{H}_{2} \mathrm{O}\right)_{1}+\mathrm{H}_{2} \mathrm{O} \Leftrightarrow\left(\mathrm{NH}_{4}^{+}\right)\left(\mathrm{H}_{2} \mathrm{SO}_{4}\right)\left(\mathrm{H}_{2} \mathrm{O}\right)_{2}$ & -17.69 & -8.11 \\
\hline$\left(\mathrm{NH}_{4}^{+}\right)\left(\mathrm{H}_{2} \mathrm{O}\right)_{1}+\mathrm{H}_{2} \mathrm{O} \Leftrightarrow\left(\mathrm{NH}_{4}^{+}\right)\left(\mathrm{H}_{2} \mathrm{O}\right)_{2}$ & $-15.83(-17.04)$ & $-10.62(-8.63)$ \\
\hline$\left(\mathrm{H}_{3} \mathrm{O}^{+}\right)\left(\mathrm{H}_{2} \mathrm{SO}_{4}\right)\left(\mathrm{H}_{2} \mathrm{O}\right)_{1}+\mathrm{H}_{2} \mathrm{O} \Leftrightarrow\left(\mathrm{H}_{3} \mathrm{O}^{+}\right)\left(\mathrm{H}_{2} \mathrm{SO}_{4}\right)\left(\mathrm{H}_{2} \mathrm{O}\right)_{2}$ & -16.84 & -10.35 \\
\hline$\left(\mathrm{H}_{3} \mathrm{O}^{+}\right)\left(\mathrm{H}_{2} \mathrm{O}\right)_{1}+\mathrm{H}_{2} \mathrm{O} \Leftrightarrow\left(\mathrm{H}_{3} \mathrm{O}^{+}\right)\left(\mathrm{H}_{2} \mathrm{O}\right)_{2}$ & -22.81 & -14.65 \\
\hline$\left(\mathrm{NH}_{3}\right)\left(\mathrm{H}_{2} \mathrm{SO}_{4}\right)\left(\mathrm{H}_{2} \mathrm{O}\right)_{1}+\mathrm{H}_{2} \mathrm{O} \Leftrightarrow\left(\mathrm{NH}_{3}\right)\left(\mathrm{H}_{2} \mathrm{SO}_{4}\right)\left(\mathrm{H}_{2} \mathrm{O}\right)_{2}$ & -14.07 & -3.05 \\
\hline$\left(\mathrm{NH}_{4}^{+}\right)\left(\mathrm{H}_{2} \mathrm{SO}_{4}\right)\left(\mathrm{H}_{2} \mathrm{O}\right)_{2}+\mathrm{H}_{2} \mathrm{O} \Leftrightarrow\left(\mathrm{NH}_{4}^{+}\right)\left(\mathrm{H}_{2} \mathrm{SO}_{4}\right)\left(\mathrm{H}_{2} \mathrm{O}\right)_{3}$ & -14.90 & -6.09 \\
\hline$\left(\mathrm{NH}_{4}^{+}\right)\left(\mathrm{H}_{2} \mathrm{O}\right)_{2}+\mathrm{H}_{2} \mathrm{O} \Leftrightarrow\left(\mathrm{NH}_{4}^{+}\right)\left(\mathrm{H}_{2} \mathrm{O}\right)_{3}$ & $-13.41(-14.71)$ & $-5.49(-5.97)$ \\
\hline$\left(\mathrm{H}_{3} \mathrm{O}^{+}\right)\left(\mathrm{H}_{2} \mathrm{SO}_{4}\right)\left(\mathrm{H}_{2} \mathrm{O}\right)_{2}+\mathrm{H}_{2} \mathrm{O} \Leftrightarrow\left(\mathrm{H}_{3} \mathrm{O}^{+}\right)\left(\mathrm{H}_{2} \mathrm{SO}_{4}\right)\left(\mathrm{H}_{2} \mathrm{O}\right)_{3}$ & -18.63 & -7.07 \\
\hline$\left(\mathrm{H}_{3} \mathrm{O}^{+}\right)\left(\mathrm{H}_{2} \mathrm{O}\right)_{2}+\mathrm{H}_{2} \mathrm{O} \Leftrightarrow\left(\mathrm{H}_{3} \mathrm{O}^{+}\right)\left(\mathrm{H}_{2} \mathrm{O}\right)_{3}$ & -18.12 & -10.36 \\
\hline$\left(\mathrm{NH}_{3}\right)\left(\mathrm{H}_{2} \mathrm{SO}_{4}\right)\left(\mathrm{H}_{2} \mathrm{O}\right)_{2}+\mathrm{H}_{2} \mathrm{O} \Leftrightarrow\left(\mathrm{NH}_{3}\right)\left(\mathrm{H}_{2} \mathrm{SO}_{4}\right)\left(\mathrm{H}_{2} \mathrm{O}\right)_{3}$ & -11.6 & -2.96 \\
\hline$\left(\mathrm{NH}_{4}^{+}\right)\left(\mathrm{H}_{2} \mathrm{SO}_{4}\right)\left(\mathrm{H}_{2} \mathrm{O}\right)_{3}+\mathrm{H}_{2} \mathrm{O} \Leftrightarrow\left(\mathrm{NH}_{4}^{+}\right)\left(\mathrm{H}_{2} \mathrm{SO}_{4}\right)\left(\mathrm{H}_{2} \mathrm{O}\right)_{4}$ & -12.04 & -4.25 \\
\hline$\left(\mathrm{NH}_{4}^{+}\right)\left(\mathrm{H}_{2} \mathrm{O}\right)_{3}+\mathrm{H}_{2} \mathrm{O} \Leftrightarrow\left(\mathrm{NH}_{4}^{+}\right)\left(\mathrm{H}_{2} \mathrm{O}\right)_{4}$ & $(-12.72)$ & $(-5.11)$ \\
\hline$\left(\mathrm{H}_{3} \mathrm{O}^{+}\right)\left(\mathrm{H}_{2} \mathrm{SO}_{4}\right)\left(\mathrm{H}_{2} \mathrm{O}\right)_{3}+\mathrm{H}_{2} \mathrm{O} \Leftrightarrow\left(\mathrm{H}_{3} \mathrm{O}^{+}\right)\left(\mathrm{H}_{2} \mathrm{SO}_{4}\right)\left(\mathrm{H}_{2} \mathrm{O}\right)_{4}$ & -14.84 & -6.65 \\
\hline$\left(\mathrm{H}_{3} \mathrm{O}^{+}\right)\left(\mathrm{H}_{2} \mathrm{O}\right)_{3}+\mathrm{H}_{2} \mathrm{O} \Leftrightarrow\left(\mathrm{H}_{3} \mathrm{O}^{+}\right)\left(\mathrm{H}_{2} \mathrm{O}\right)_{4}$ & -13.65 & -5.54 \\
\hline$\left(\mathrm{NH}_{3}\right)\left(\mathrm{H}_{2} \mathrm{SO}_{4}\right)\left(\mathrm{H}_{2} \mathrm{O}\right)_{3}+\mathrm{H}_{2} \mathrm{O} \Leftrightarrow\left(\mathrm{NH}_{3}\right)\left(\mathrm{H}_{2} \mathrm{SO}_{4}\right)\left(\mathrm{H}_{2} \mathrm{O}\right)_{4}$ & -10.77 & -2.28 \\
\hline$\left(\mathrm{NH}_{4}^{+}\right)\left(\mathrm{H}_{2} \mathrm{SO}_{4}\right)\left(\mathrm{H}_{2} \mathrm{O}\right)_{4}+\mathrm{H}_{2} \mathrm{O} \Leftrightarrow\left(\mathrm{NH}_{4}^{+}\right)\left(\mathrm{H}_{2} \mathrm{SO}_{4}\right)\left(\mathrm{H}_{2} \mathrm{O}\right)_{5}$ & -11.68 & -1.63 \\
\hline$\left(\mathrm{NH}_{4}^{+}\right)\left(\mathrm{H}_{2} \mathrm{O}\right)_{4}+\mathrm{H}_{2} \mathrm{O} \Leftrightarrow\left(\mathrm{NH}_{4}^{+}\right)\left(\mathrm{H}_{2} \mathrm{O}\right)_{5}^{+}$ & -10.89 & -0.31 \\
\hline$\left(\mathrm{H}_{3} \mathrm{O}^{+}\right)\left(\mathrm{H}_{2} \mathrm{SO}_{4}\right)\left(\mathrm{H}_{2} \mathrm{O}\right)_{4}+\mathrm{H}_{2} \mathrm{O} \Leftrightarrow\left(\mathrm{H}_{3} \mathrm{O}^{+}\right)\left(\mathrm{H}_{2} \mathrm{SO}_{4}\right)\left(\mathrm{H}_{2} \mathrm{O}\right)_{5}$ & -12.48 & -4.47 \\
\hline$\left(\mathrm{H}_{3} \mathrm{O}^{+}\right)\left(\mathrm{H}_{2} \mathrm{O}\right)_{4}+\mathrm{H}_{2} \mathrm{O} \Leftrightarrow\left(\mathrm{H}_{3} \mathrm{O}^{+}\right)\left(\mathrm{H}_{2} \mathrm{O}\right)_{5}$ & -12.08 & -4.17 \\
\hline$\left(\mathrm{NH}_{3}\right)\left(\mathrm{H}_{2} \mathrm{SO}_{4}\right)\left(\mathrm{H}_{2} \mathrm{O}\right)_{4}+\mathrm{H}_{2} \mathrm{O} \Leftrightarrow\left(\mathrm{NH}_{3}\right)\left(\mathrm{H}_{2} \mathrm{SO}_{4}\right)\left(\mathrm{H}_{2} \mathrm{O}\right)_{5}$ & -10.7 & -0.01 \\
\hline$\left(\mathrm{NH}_{4}^{+}\right)\left(\mathrm{H}_{2} \mathrm{SO}_{4}\right)\left(\mathrm{H}_{2} \mathrm{O}\right)_{5}+\mathrm{H}_{2} \mathrm{O} \Leftrightarrow\left(\mathrm{NH}_{4}^{+}\right)\left(\mathrm{H}_{2} \mathrm{SO}_{4}\right)\left(\mathrm{H}_{2} \mathrm{O}\right)_{6}$ & -12.78 & -2.44 \\
\hline$\left(\mathrm{NH}_{4}^{+}\right)\left(\mathrm{H}_{2} \mathrm{O}\right)_{5}+\mathrm{H}_{2} \mathrm{O} \Leftrightarrow\left(\mathrm{NH}_{4}^{+}\right)\left(\mathrm{H}_{2} \mathrm{O}\right)_{6}$ & -12.45 & -3.52 \\
\hline$\left(\mathrm{H}_{3} \mathrm{O}^{+}\right)\left(\mathrm{H}_{2} \mathrm{SO}_{4}\right)\left(\mathrm{H}_{2} \mathrm{O}\right)_{5}+\mathrm{H}_{2} \mathrm{O} \Leftrightarrow\left(\mathrm{H}_{3} \mathrm{O}^{+}\right)\left(\mathrm{H}_{2} \mathrm{SO}_{4}\right)\left(\mathrm{H}_{2} \mathrm{O}\right)_{6}$ & -14.2 & -1.9 \\
\hline$\left(\mathrm{H}_{3} \mathrm{O}^{+}\right)\left(\mathrm{H}_{2} \mathrm{O}\right)_{5}+\mathrm{H}_{2} \mathrm{O} \Leftrightarrow\left(\mathrm{H}_{3} \mathrm{O}^{+}\right)\left(\mathrm{H}_{2} \mathrm{O}\right)_{6}$ & -11.16 & -3.50 \\
\hline$\left(\mathrm{NH}_{4}^{+}\right)\left(\mathrm{H}_{2} \mathrm{O}\right)_{6}+\mathrm{H}_{2} \mathrm{O} \Leftrightarrow\left(\mathrm{NH}_{4}^{+}\right)\left(\mathrm{H}_{2} \mathrm{O}\right)_{7}$ & -11.03 & 0.87 \\
\hline$\left(\mathrm{H}_{3} \mathrm{O}^{+}\right)\left(\mathrm{H}_{2} \mathrm{O}\right)_{6}+\mathrm{H}_{2} \mathrm{O} \Leftrightarrow\left(\mathrm{H}_{3}^{+} \mathrm{O}^{+}\right)\left(\mathrm{H}_{2} \mathrm{O}\right)_{7}$ & -9.79 & -2.46 \\
\hline
\end{tabular}

$\left(\mathrm{NH}_{3}\right)\left(\mathrm{H}_{2} \mathrm{SO}_{4}\right)\left(\mathrm{H}_{2} \mathrm{O}\right)_{n}$, and $\left(\mathrm{H}_{3} \mathrm{O}\right)^{+}\left(\mathrm{H}_{2} \mathrm{O}\right)_{n-1}+\mathrm{NH}_{3} \leftrightarrow$ $\left(\mathrm{NH}_{4}\right)^{+}\left(\mathrm{H}_{2} \mathrm{O}\right)_{n}$. It is clear from Table 3 that the conversion of $\left(\mathrm{H}_{3} \mathrm{O}\right)^{+}\left(\mathrm{H}_{2} \mathrm{O}\right)_{n-1}$ into $\left(\mathrm{NH}_{4}\right)^{+}\left(\mathrm{H}_{2} \mathrm{O}\right)_{n}$ and $\left(\mathrm{H}_{3} \mathrm{O}\right)^{+}\left(\mathrm{H}_{2} \mathrm{SO}_{4}\right)\left(\mathrm{H}_{2} \mathrm{O}\right)_{n}$ into $\left(\mathrm{NH}_{4}\right)^{+}\left(\mathrm{H}_{2} \mathrm{SO}_{4}\right)\left(\mathrm{H}_{2} \mathrm{O}\right)_{n+1}$ are favorable thermodynamically in all the cases studied here. The high $\left(\sim 13-33 \mathrm{kcal} \mathrm{mole}^{-1}\right)$ and $\left(\sim 10-52 \mathrm{kcal} \mathrm{mole}^{-1}\right)$ affinities of ammonia to $\left(\mathrm{H}_{3} \mathrm{O}\right)^{+}\left(\mathrm{H}_{2} \mathrm{SO}_{4}\right)\left(\mathrm{H}_{2} \mathrm{O}\right)_{n-1}$ and $\left(\mathrm{H}_{3} \mathrm{O}\right)^{+}\left(\mathrm{H}_{2} \mathrm{O}\right)_{n-1}$, respectively, gives us a clear indication that in the real atmospheric conditions these reactions are kinetically controlled. We also note that the affinities of ammonia to $\left(\mathrm{H}_{3} \mathrm{O}\right)^{+}\left(\mathrm{H}_{2} \mathrm{SO}_{4}\right)\left(\mathrm{H}_{2} \mathrm{O}\right)_{n-1}$ and $\left(\mathrm{H}_{3} \mathrm{O}\right)^{+}\left(\mathrm{H}_{2} \mathrm{O}\right)_{n-1}$ decrease with hydration number $(n)$, suggesting that the relative abundance of positive ion clusters containing hydronium $\left(\mathrm{H}_{3} \mathrm{O}^{+}\right)$and those containing protonated ammonia depends not only on ammonia concentration but also on relative humidity.
Figure 3 compares the affinities of ammonia to $\left(\mathrm{H}_{3} \mathrm{O}\right)^{+}\left(\mathrm{H}_{2} \mathrm{SO}_{4}\right)\left(\mathrm{H}_{2} \mathrm{O}\right)_{n}$ (obtained in the present study) with those to $\left(\mathrm{H}_{2} \mathrm{SO}_{4}\right)\left(\mathrm{H}_{2} \mathrm{O}\right)_{n}$ and $\left(\mathrm{HSO}_{4}^{-}\right)\left(\mathrm{H}_{2} \mathrm{O}\right)_{n}$ (from previous studies). The affinity of ammonia to positively charged, negatively charged, and neutral sulfuric acid clusters differ significantly. While hydration number $(n)$ has small effect on the affinity of ammonia to neutral and negatively charged clusters, it has much stronger effect on the affinity of ammonia to positively charged cluster. The affinities of ammonia to positively charged clusters are much higher than those to neutral and negative clusters of similar composition, and the difference for $n=0-5$ reaches $7-25 \mathrm{kcal} \mathrm{mole}^{-1}$ and $13-$ $34 \mathrm{kcal} \mathrm{mole}^{-1}$, respectively. 
Table 2. Comparison of the changes in enthalpies and Gibbs free energies ( $\mathrm{kcalmole}^{-1}$ ) associated with the formation of $\left(\mathrm{NH}_{4}^{+}\right)\left(\mathrm{H}_{2} \mathrm{SO}_{4}\right)\left(\mathrm{H}_{2} \mathrm{O}\right)_{n}$ from $\left(\mathrm{H}_{3} \mathrm{O}^{+}\right)\left(\mathrm{H}_{2} \mathrm{SO}_{4}\right)\left(\mathrm{H}_{2} \mathrm{O}\right)_{n}$ by the addition of the sulfuric acid. $T=298.15 \mathrm{~K}$ and $P=101.3 \mathrm{KPa}$ unless specified. In the last column, the numbers in the parentheses are from Froyd and Lovejoy (2003).

\begin{tabular}{lrrr}
\hline & $\Delta \mathrm{H}$ & $\Delta \mathrm{Gg} \Delta \mathrm{G}$ at $270 \mathrm{~K}$ & \\
\hline$\left(\mathrm{H}_{3} \mathrm{O}^{+}\right)+\left(\mathrm{H}_{2} \mathrm{SO}_{4}\right) \Leftrightarrow\left(\mathrm{H}_{3} \mathrm{O}^{+}\right)\left(\mathrm{H}_{2} \mathrm{SO}_{4}\right)$ & -37.38 & -28.21 & $-29.07(-25.5 \pm 2)$ \\
$\left(\mathrm{NH}_{4}^{+}\right)\left(\mathrm{H}_{2} \mathrm{O}\right)_{1}+\left(\mathrm{H}_{2} \mathrm{SO}_{4}\right) \Leftrightarrow\left(\mathrm{NH}_{4}^{+}\right)\left(\mathrm{H}_{2} \mathrm{SO}_{4}\right)\left(\mathrm{H}_{2} \mathrm{O}\right)_{1}$ & -17.55 & -9.74 & \\
$\left(\mathrm{H}_{3} \mathrm{O}^{+}\right)\left(\mathrm{H}_{2} \mathrm{O}\right)_{1}+\left(\mathrm{H}_{2} \mathrm{SO}_{4}\right) \Leftrightarrow\left(\mathrm{H}_{3} \mathrm{O}^{+}\right)\left(\mathrm{H}_{2} \mathrm{SO}_{4}\right)\left(\mathrm{H}_{2} \mathrm{O}\right)_{1}$ & -24.02 & -13.62 & $-14.6(-14.3 \pm 2)$ \\
$\left(\mathrm{NH}_{4}^{+}\right)\left(\mathrm{H}_{2} \mathrm{O}\right)_{2}+\left(\mathrm{H}_{2} \mathrm{SO}_{4}\right) \Leftrightarrow\left(\mathrm{NH}_{4}^{+}\right)\left(\mathrm{H}_{2} \mathrm{SO}_{4}\right)\left(\mathrm{H}_{2} \mathrm{O}\right)_{2}$ & -19.41 & -7.23 & \\
$\left(\mathrm{H}_{3} \mathrm{O}^{+}\right)\left(\mathrm{H}_{2} \mathrm{O}\right)_{2}+\left(\mathrm{H}_{2} \mathrm{SO}_{4}\right) \Leftrightarrow\left(\mathrm{H}_{3} \mathrm{O}^{+}\right)\left(\mathrm{H}_{2} \mathrm{SO}_{4}\right)\left(\mathrm{H}_{2} \mathrm{O}\right)_{2}$ & -18.05 & -9.32 & $-10.1(-12.5 \pm 2)$ \\
$\left(\mathrm{NH}_{4}^{+}\right)\left(\mathrm{H}_{2} \mathrm{O}\right)_{3}+\left(\mathrm{H}_{2} \mathrm{SO}_{4}\right) \Leftrightarrow\left(\mathrm{NH}_{4}^{+}\right)\left(\mathrm{H}_{2} \mathrm{SO}_{4}\right)\left(\mathrm{H}_{2} \mathrm{O}\right)_{3}$ & -20.90 & -7.84 & \\
$\left(\mathrm{H}_{3} \mathrm{O}^{+}\right)\left(\mathrm{H}_{2} \mathrm{O}\right)_{3}+\left(\mathrm{H}_{2} \mathrm{SO}_{4}\right) \Leftrightarrow\left(\mathrm{H}_{3} \mathrm{O}^{+}\right)\left(\mathrm{H}_{2} \mathrm{SO}_{4}\right)\left(\mathrm{H}_{2} \mathrm{O}\right)_{3}$ & -14.77 & -5.61 & $-6.5(-10.7 \pm 2)$ \\
$\left(\mathrm{NH}_{4}^{+}\right)\left(\mathrm{H}_{2} \mathrm{O}\right)_{4}+\left(\mathrm{H}_{2} \mathrm{SO}_{4}\right) \Leftrightarrow\left(\mathrm{NH}_{4}^{+}\right)\left(\mathrm{H}_{2} \mathrm{SO}_{4}\right)\left(\mathrm{H}_{2} \mathrm{O}\right)_{4}$ & -21.85 & -6.21 & \\
$\left(\mathrm{H}_{3} \mathrm{O}^{+}\right)\left(\mathrm{H}_{2} \mathrm{O}\right)_{4}+\left(\mathrm{H}_{2} \mathrm{SO}_{4}\right) \Leftrightarrow\left(\mathrm{H}_{3} \mathrm{O}^{+}\right)\left(\mathrm{H}_{2} \mathrm{SO}_{4}\right)\left(\mathrm{H}_{2} \mathrm{O}\right)_{4}$ & -19.75 & -7.15 & $-8.3(-10.9 \pm 2)$ \\
$\left(\mathrm{NH}_{4}^{+}\right)\left(\mathrm{H}_{2} \mathrm{O}\right)_{5}+\left(\mathrm{H}_{2} \mathrm{SO}_{4}\right) \Leftrightarrow\left(\mathrm{NH}_{4}^{+}\right)\left(\mathrm{H}_{2} \mathrm{SO}_{4}\right)\left(\mathrm{H}_{2} \mathrm{O}\right)_{5}$ & -25.09 & -7.82 & \\
$\left(\mathrm{H}_{3} \mathrm{O}^{+}\right)\left(\mathrm{H}_{2} \mathrm{O}\right)_{5}+\left(\mathrm{H}_{2} \mathrm{SO}_{4}\right) \Leftrightarrow\left(\mathrm{H}_{3} \mathrm{O}^{+}\right)\left(\mathrm{H}_{2} \mathrm{SO}_{4}\right)\left(\mathrm{H}_{2} \mathrm{O}\right)_{5}$ & -20.15 & -7.45 & $-8.6(-10.9 \pm 2)$ \\
$\left(\mathrm{NH}_{4}^{+}\right)\left(\mathrm{H}_{2} \mathrm{O}\right)_{6}+\left(\mathrm{H}_{2} \mathrm{SO}_{4}\right) \Leftrightarrow\left(\mathrm{NH}_{4}^{+}\right)\left(\mathrm{H}_{2} \mathrm{SO}_{4}\right)\left(\mathrm{H}_{2} \mathrm{O}\right)_{6}$ & -25.31 & -6.33 & \\
$\left(\mathrm{H}_{3} \mathrm{O}^{+}\right)\left(\mathrm{H}_{2} \mathrm{O}\right)_{6}+\left(\mathrm{H}_{2} \mathrm{SO}_{4}\right) \Leftrightarrow\left(\mathrm{H}_{3} \mathrm{O}^{+}\right)\left(\mathrm{H}_{2} \mathrm{SO}_{4}\right)\left(\mathrm{H}_{2} \mathrm{O}\right)_{6}$ & -24.70 & -6.59 & $-8.3(-11.1 \pm 2)$ \\
\hline
\end{tabular}

Table 3. Enthalpy and Gibbs free energy changes $(\mathrm{kcal} \mathrm{mole}-1)$ associated with $\left(\mathrm{H}_{3} \mathrm{O}\right)^{+}\left(\mathrm{H}_{2} \mathrm{SO}_{4}\right)\left(\mathrm{H}_{2} \mathrm{O}\right)_{n-1}+\mathrm{NH}_{3} \leftrightarrow\left(\mathrm{NH}_{4}\right)^{+}\left(\mathrm{H}_{2} \mathrm{SO}_{4}\right)\left(\mathrm{H}_{2} \mathrm{O}\right)_{n}$, $\left(\mathrm{H}_{2} \mathrm{SO}_{4}\right)\left(\mathrm{H}_{2} \mathrm{O}\right)_{n}+\mathrm{NH}_{3} \leftrightarrow\left(\mathrm{NH}_{3}\right)\left(\mathrm{H}_{2} \mathrm{SO}_{4}\right)\left(\mathrm{H}_{2} \mathrm{O}\right)_{n}$ and $\left(\mathrm{H}_{3} \mathrm{O}\right)^{+}\left(\mathrm{H}_{2} \mathrm{O}\right)_{n-1}+\mathrm{NH}_{3} \leftrightarrow\left(\mathrm{NH}_{4}\right)^{+}\left(\mathrm{H}_{2} \mathrm{O}\right)_{n}$ reactions. $T=298.15 \mathrm{~K}$ and $P=101.3 \mathrm{KPa}$.

\begin{tabular}{|c|c|c|}
\hline \multicolumn{3}{|c|}{$\Delta \mathrm{H}$} \\
\hline$\left(\mathrm{H}_{2} \mathrm{SO}_{4}\right)\left(\mathrm{H}_{2} \mathrm{O}\right)_{0}+\mathrm{NH}_{3} \leftrightarrow\left(\mathrm{NH}_{3}\right)\left(\mathrm{H}_{2} \mathrm{SO}_{4}\right)\left(\mathrm{H}_{2} \mathrm{O}\right)_{0}$ & -16.71 & -7.77 \\
\hline$\left(\mathrm{H}_{3} \mathrm{O}\right)^{+}\left(\mathrm{H}_{2} \mathrm{SO}_{4}\right)\left(\mathrm{H}_{2} \mathrm{O}\right)_{0}+\mathrm{NH}_{3} \leftrightarrow\left(\mathrm{NH}_{4}\right)^{+}\left(\mathrm{H}_{2} \mathrm{SO}_{4}\right)\left(\mathrm{H}_{2} \mathrm{O}\right)_{1}$ & -40.59 & -33.52 \\
\hline$\left(\mathrm{H}_{2} \mathrm{SO}_{4}\right)\left(\mathrm{H}_{2} \mathrm{O}\right)_{1}+\mathrm{NH}_{3} \leftrightarrow\left(\mathrm{NH}_{3}\right)\left(\mathrm{H}_{2} \mathrm{SO}_{4}\right)\left(\mathrm{H}_{2} \mathrm{O}\right)_{1}$ & -15.91 & -6.9 \\
\hline$\left(\mathrm{H}_{3} \mathrm{O}\right)^{+}\left(\mathrm{H}_{2} \mathrm{O}\right)_{0}+\mathrm{NH}_{3} \leftrightarrow\left(\mathrm{NH}_{4}\right)^{+}\left(\mathrm{H}_{2} \mathrm{O}\right)_{1}$ & -60.45 & -52.01 \\
\hline$\left(\mathrm{H}_{3} \mathrm{O}\right)^{+}\left(\mathrm{H}_{2} \mathrm{SO}_{4}\right)\left(\mathrm{H}_{2} \mathrm{O}\right)_{1}+\mathrm{NH}_{3} \leftrightarrow\left(\mathrm{NH}_{4}\right)^{+}\left(\mathrm{H}_{2} \mathrm{SO}_{4}\right)\left(\mathrm{H}_{2} \mathrm{O}\right)_{2}$ & -33.82 & -27.35 \\
\hline$\left(\mathrm{H}_{2} \mathrm{SO}_{4}\right)\left(\mathrm{H}_{2} \mathrm{O}\right)_{2}+\mathrm{NH}_{3} \leftrightarrow\left(\mathrm{NH}_{3}\right)\left(\mathrm{H}_{2} \mathrm{SO}_{4}\right)\left(\mathrm{H}_{2} \mathrm{O}\right)_{2}$ & -17.42 & -6.94 \\
\hline$\left(\mathrm{H}_{3} \mathrm{O}\right)^{+}\left(\mathrm{H}_{2} \mathrm{O}\right)_{1}+\mathrm{NH}_{3} \leftrightarrow\left(\mathrm{NH}_{4}\right)^{+}\left(\mathrm{H}_{2} \mathrm{O}\right)_{2}$ & -38.44 & -33.73 \\
\hline$\left(\mathrm{H}_{3} \mathrm{O}\right)^{+}\left(\mathrm{H}_{2} \mathrm{SO}_{4}\right)\left(\mathrm{H}_{2} \mathrm{O}\right)_{2}+\mathrm{NH}_{3} \leftrightarrow\left(\mathrm{NH}_{4}\right)^{+}\left(\mathrm{H}_{2} \mathrm{SO}_{4}\right)\left(\mathrm{H}_{2} \mathrm{O}\right)_{3}$ & -31.88 & -23.09 \\
\hline$\left(\mathrm{H}_{2} \mathrm{SO}_{4}\right)\left(\mathrm{H}_{2} \mathrm{O}\right)_{3}+\mathrm{NH}_{3} \leftrightarrow\left(\mathrm{NH}_{3}\right)\left(\mathrm{H}_{2} \mathrm{SO}_{4}\right)\left(\mathrm{H}_{2} \mathrm{O}\right)_{3}$ & -17.68 & -8.01 \\
\hline$\left(\mathrm{H}_{3} \mathrm{O}\right)^{+}\left(\mathrm{H}_{2} \mathrm{O}\right)_{2}+\mathrm{NH}_{3} \leftrightarrow\left(\mathrm{NH}_{4}\right)^{+}\left(\mathrm{H}_{2} \mathrm{O}\right)_{3}$ & -29.03 & -24.57 \\
\hline$\left(\mathrm{H}_{3} \mathrm{O}\right)^{+}\left(\mathrm{H}_{2} \mathrm{SO}_{4}\right)\left(\mathrm{H}_{2} \mathrm{O}\right)_{3}+\mathrm{NH}_{3} \leftrightarrow\left(\mathrm{NH}_{4}\right)^{+}\left(\mathrm{H}_{2} \mathrm{SO}_{4}\right)\left(\mathrm{H}_{2} \mathrm{O}\right)_{4}$ & -29.19 & -20.72 \\
\hline$\left(\mathrm{H}_{2} \mathrm{SO}_{4}\right)\left(\mathrm{H}_{2} \mathrm{O}\right)_{4}+\mathrm{NH}_{3} \leftrightarrow\left(\mathrm{NH}_{3}\right)\left(\mathrm{H}_{2} \mathrm{SO}_{4}\right)\left(\mathrm{H}_{2} \mathrm{O}\right)_{4}$ & -13.96 & -6.88 \\
\hline$\left(\mathrm{H}_{3} \mathrm{O}\right)^{+}\left(\mathrm{H}_{2} \mathrm{O}\right)_{3}+\mathrm{NH}_{3} \leftrightarrow\left(\mathrm{NH}_{4}\right)^{+}\left(\mathrm{H}_{2} \mathrm{O}\right)_{4}$ & -21.99 & -20.09 \\
\hline$\left(\mathrm{H}_{3} \mathrm{O}\right)^{+}\left(\mathrm{H}_{2} \mathrm{SO}_{4}\right)\left(\mathrm{H}_{2} \mathrm{O}\right)_{4}+\mathrm{NH}_{3} \leftrightarrow\left(\mathrm{NH}_{4}\right)^{+}\left(\mathrm{H}_{2} \mathrm{SO}_{4}\right)\left(\mathrm{H}_{2} \mathrm{O}\right)_{5}$ & -22.24 & -15.28 \\
\hline$\left(\mathrm{H}_{2} \mathrm{SO}_{4}\right)\left(\mathrm{H}_{2} \mathrm{O}\right)_{5}+\mathrm{NH}_{3} \leftrightarrow\left(\mathrm{NH}_{3}\right)\left(\mathrm{H}_{2} \mathrm{SO}_{4}\right)\left(\mathrm{H}_{2} \mathrm{O}\right)_{5}$ & -14.66 & -5.66 \\
\hline$\left(\mathrm{H}_{3} \mathrm{O}\right)^{+}\left(\mathrm{H}_{2} \mathrm{O}\right)_{4}+\mathrm{NH}_{3} \leftrightarrow\left(\mathrm{NH}_{4}\right)^{+}\left(\mathrm{H}_{2} \mathrm{O}\right)_{5}$ & -19.23 & -14.86 \\
\hline $\begin{array}{l}\left(\mathrm{H}_{3} \mathrm{O}\right)^{+}\left(\mathrm{H}_{2} \mathrm{SO}_{4}\right)\left(\mathrm{H}_{2} \mathrm{O}\right)_{5}+\mathrm{NH}_{3} \leftrightarrow\left(\mathrm{NH}_{4}\right)^{+}\left(\mathrm{H}_{2} \mathrm{SO}_{4}\right)\left(\mathrm{H}_{2} \mathrm{O}\right)_{6} \\
\left(\mathrm{H}_{2} \mathrm{SO}_{4}\right)\left(\mathrm{H}_{2} \mathrm{O}\right)_{6}+\mathrm{NH}_{3} \leftrightarrow\left(\mathrm{NH}_{3}\right)\left(\mathrm{H}_{2} \mathrm{SO}_{4}\right)\left(\mathrm{H}_{2} \mathrm{O}\right)_{6}\end{array}$ & -22.54 & -13.25 \\
\hline$\left(\mathrm{H}_{3} \mathrm{O}\right)^{+}\left(\mathrm{H}_{2} \mathrm{O}\right)_{5}+\mathrm{NH}_{3} \leftrightarrow\left(\mathrm{NH}_{4}\right)^{+}\left(\mathrm{H}_{2} \mathrm{O}\right)_{6}$ & -19.6 & -14.22 \\
\hline
\end{tabular}

Based on the thermochemical quantities presented above, we can calculate the abundance of charged and neutral clusters containing ammonia under typical atmospheric conditions. Figure 4 presents the equilibrium concentration ratios of ternary (ammonized) and binary clusters containing different number of water molecules under two sets of atmospheric conditions $\left(T=295 \mathrm{~K},\left[\mathrm{NH}_{3}\right]=1 \mathrm{ppb}\right.$ and $T=270 \mathrm{~K}$,
$\left.\left[\mathrm{NH}_{3}\right]=0.1 \mathrm{ppb}\right)$. It is clear from Fig. 4 that the hydrated negative monomers of the sulfuric acid contain no ammonia and that less than $\sim 0.1 \%$ of neutral hydrated sulfuric acid monomers contain ammonia, which are consistent with previous studies of Kurten et al. (2008a, b) and Ortega et al. (2008). In contrast, most of positively charged hydrated sulfuric acid monomers appear to be ammonized 


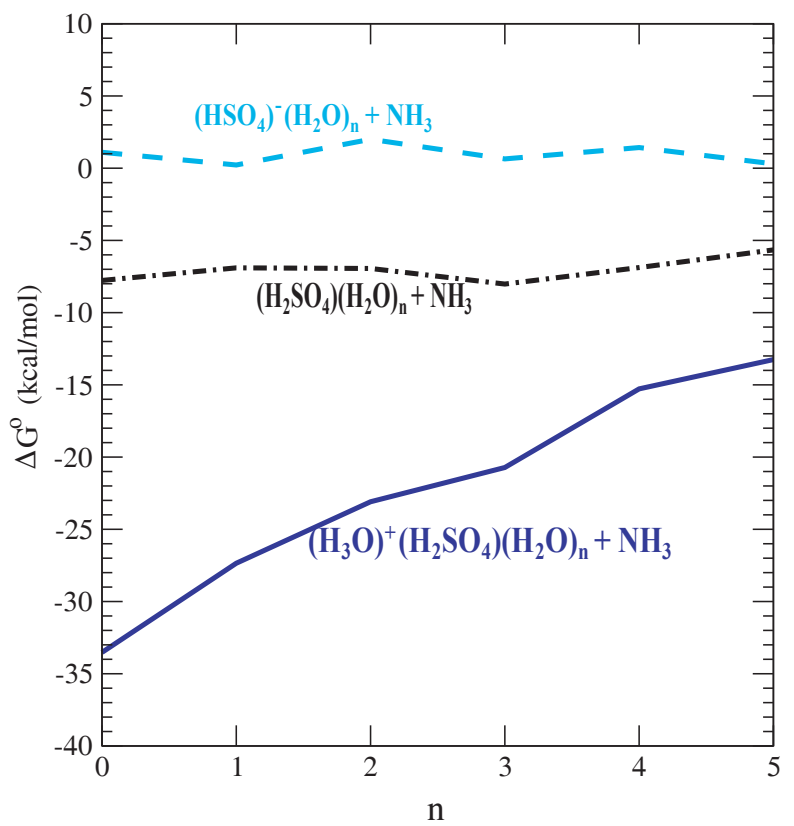

Fig. 3. A comparison of the affinity of ammonia to positive, neutral, and negative hydrated sulfuric acid monomers containing 1-5 water molecules. The data for neutral clusters are from Nadykto et al. (2008a) and the data for negative ions are from Nadykto et al. (2008b).

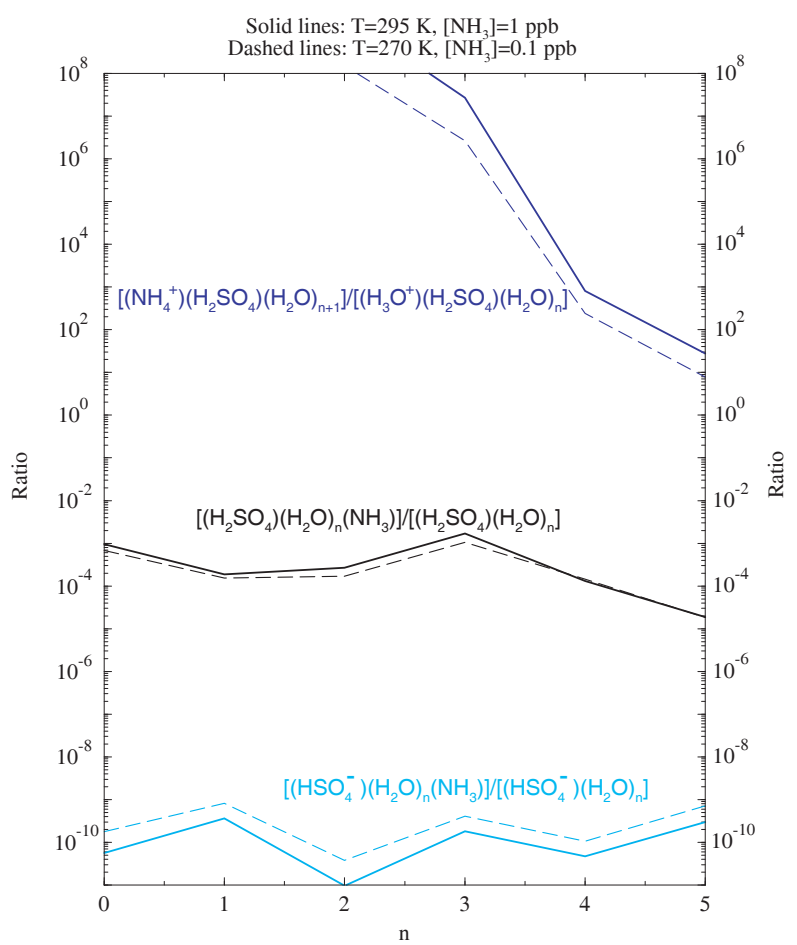

Fig. 4. The ratio of equilibrium concentrations of ternary (ammonized) and binary clusters containing different number of water molecules $(n)$, under two sets of atmospheric conditions.

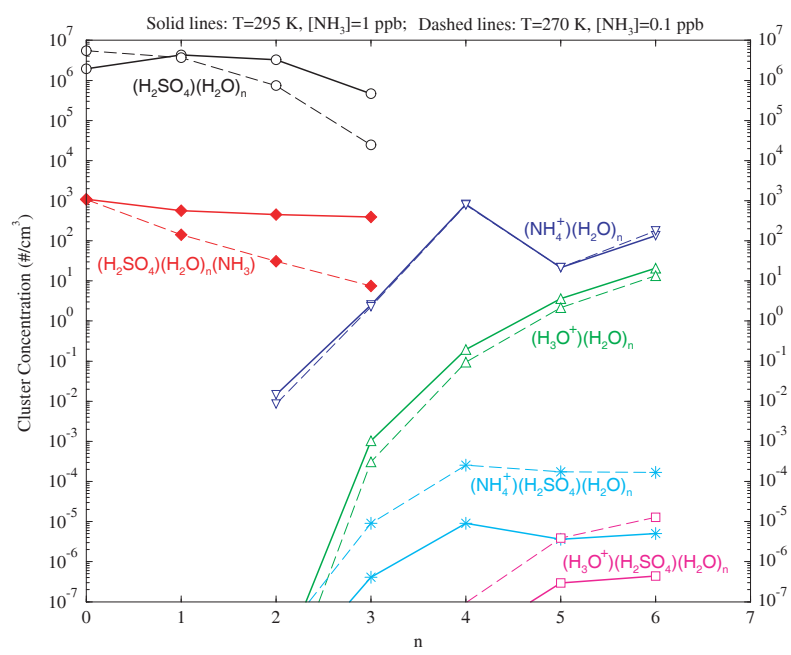

Fig. 5. The equilibrium concentrations of $\left(\mathrm{H}_{2} \mathrm{SO}_{4}\right)\left(\mathrm{H}_{2} \mathrm{O}\right)_{n}$, $\left(\mathrm{NH}_{3}\right)\left(\mathrm{H}_{2} \mathrm{SO}_{4}\right)\left(\mathrm{H}_{2} \mathrm{O}\right)_{n}, \quad\left(\mathrm{H}_{3} \mathrm{O}\right)^{+}\left(\mathrm{H}_{2} \mathrm{O}\right)_{n-1}, \quad\left(\mathrm{H}_{3} \mathrm{O}\right)^{+}\left(\mathrm{H}_{2} \mathrm{SO}_{4}\right)$ $\left(\mathrm{H}_{2} \mathrm{O}\right)_{n}, \quad\left(\mathrm{NH}_{4}\right)^{+}\left(\mathrm{H}_{2} \mathrm{O}\right)_{n}$ and $\left(\mathrm{NH}_{4}\right)^{+}\left(\mathrm{H}_{2} \mathrm{SO}_{4}\right)\left(\mathrm{H}_{2} \mathrm{O}\right)_{n}$ under two atmospheric conditions (solid lines: $T=295 \mathrm{~K},\left[\mathrm{NH}_{3}\right]=1 \mathrm{ppb}$; dashed lines: $T=270 \mathrm{~K},\left[\mathrm{NH}_{3}\right]=0.1 \mathrm{ppb}$ ). Relative humidity of $50 \%$ is assumed. The concentrations of neutral clusters have been normalized to a total concentration of $10^{7} \mathrm{~cm}^{-3}$ and those of ionic clusters have been normalized to a total small positive ion concentration of $10^{3} \mathrm{~cm}^{-3}$, corresponding to typical concentrations observed during the nucleation periods in boreal forests.

under the given conditions. It is important to note that the ratio of $\left(\mathrm{NH}_{4}\right)^{+}\left(\mathrm{H}_{2} \mathrm{SO}_{4}\right)\left(\mathrm{H}_{2} \mathrm{O}\right)_{n+1}$ to $\left(\mathrm{H}_{3} \mathrm{O}\right)^{+}\left(\mathrm{H}_{2} \mathrm{SO}_{4}\right)\left(\mathrm{H}_{2} \mathrm{O}\right)_{n}$ decreases significantly with the hydration number.

Figure 4 gives the relative abundance of ternary and binary clusters. To assess the implications to atmospheric nucleation, it is important to know that absolute concentrations of clusters under atmospheric conditions. Figure 5 shows the equilibrium concentrations of $\left(\mathrm{H}_{2} \mathrm{SO}_{4}\right)\left(\mathrm{H}_{2} \mathrm{O}\right)_{n}$, $\left(\mathrm{NH}_{3}\right)\left(\mathrm{H}_{2} \mathrm{SO}_{4}\right)\left(\mathrm{H}_{2} \mathrm{O}\right)_{n},\left(\mathrm{H}_{3} \mathrm{O}\right)^{+}\left(\mathrm{H}_{2} \mathrm{O}\right)_{n-1},\left(\mathrm{NH}_{4}\right)^{+}\left(\mathrm{H}_{2} \mathrm{O}\right)_{n}$, $\left(\mathrm{H}_{3} \mathrm{O}\right)^{+}\left(\mathrm{H}_{2} \mathrm{SO}_{4}\right)\left(\mathrm{H}_{2} \mathrm{O}\right)_{n}$, and $\left(\mathrm{NH}_{4}\right)^{+}\left(\mathrm{H}_{2} \mathrm{SO}_{4}\right)\left(\mathrm{H}_{2} \mathrm{O}\right)_{n}$ under two atmospheric conditions, calculated based on the thermodynamic data presented in Tables $1-3$. It is clear from Fig. 5 that $\sim 10^{3} \mathrm{~cm}^{-3}$ out of $10^{7} \mathrm{~cm}^{-3}$ sulfuric acid momoners contain ammonia, consistent with the ratios shown in Fig. 4. Based on our calculations, $\left(\mathrm{NH}_{4}\right)^{+}\left(\mathrm{H}_{2} \mathrm{O}\right)_{n}$ clusters have concentrations comparable to those of $\left(\mathrm{NH}_{3}\right)\left(\mathrm{H}_{2} \mathrm{SO}_{4}\right)\left(\mathrm{H}_{2} \mathrm{O}\right)_{n}$ and appear to dominate the small positive ions. As a result of weak affinity of $\mathrm{H}_{2} \mathrm{SO}_{4}$ molecules with $\left(\mathrm{H}_{3} \mathrm{O}\right)^{+}\left(\mathrm{H}_{2} \mathrm{O}\right)_{n-1}$ and $\left(\mathrm{NH}_{4}\right)^{+}\left(\mathrm{H}_{2} \mathrm{O}\right)_{n}$, both $\left(\mathrm{NH}_{4}\right)^{+}\left(\mathrm{H}_{2} \mathrm{SO}_{4}\right)\left(\mathrm{H}_{2} \mathrm{O}\right)_{n}$ and $\left(\mathrm{H}_{3} \mathrm{O}\right)^{+}\left(\mathrm{H}_{2} \mathrm{SO}_{4}\right)\left(\mathrm{H}_{2} \mathrm{O}\right)_{n}$ clusters for $n \leq 6$ have quite low concentrations. This is consistent with the conclusion of Froyd and Lovejoy (2003) that water tends to displace sulfuric acid from small protonated sulfuric acid-water clusters. 
The low concentrations of positive sulfuric acid-water cluster ions (with $\mathrm{H}_{3} \mathrm{O}^{+}$or $\mathrm{NH}_{4}^{+}$core ions) suggests that growth of small positive ions by uptaking $\mathrm{H}_{2} \mathrm{SO}_{4}$ molecules may be insufficient. However, long-term (3 years) ion mobility measurements in the boreal forests show significant growth of both positive and negative ion clusters during the nucleation periods in many of nucleation event days (Hirsikko et al., 2007). These nucleation events and hence growth of small ions have been shown to be closely associated with the increase in $\mathrm{H}_{2} \mathrm{SO}_{4}$ vapor concentrations during the nucleation periods (Riipinen et al., 2007). The observed growth of positive ions reported in Hirsikko et al. (2007) and lower affinity of $\mathrm{H}_{2} \mathrm{SO}_{4}$ molecules to ammoniated and un-ammoniated water proton clusters suggests that the compositions of small ions in the boreal forests may be different from what have been studied here. Further research is needed to identify the possible species (such as organics, amines, etc.) in small positive ions and to understand how the changes in the ion compositions may affect $\mathrm{H}_{2} \mathrm{SO}_{4}$ affinity. It should be noted that current study of $\left(\mathrm{H}_{3} \mathrm{O}\right)^{+}\left(\mathrm{H}_{2} \mathrm{SO}_{4}\right)\left(\mathrm{H}_{2} \mathrm{O}\right)_{n}$ and $\left(\mathrm{NH}_{4}\right)^{+}\left(\mathrm{H}_{2} \mathrm{SO}_{4}\right)\left(\mathrm{H}_{2} \mathrm{O}\right)_{n}$ is limited to $n \leq 6$. Under typical boundary layer atmospheric conditions, $\left(\mathrm{H}_{3} \mathrm{O}\right)^{+}\left(\mathrm{H}_{2} \mathrm{SO}_{4}\right)\left(\mathrm{H}_{2} \mathrm{O}\right)_{n}$ distributions peak above $n=6$ because of the strong hydration of positive ions. It remains to be studied if the affinity of $\mathrm{H}_{2} \mathrm{SO}_{4}$ molecules to ammoniated and un-ammoniated water proton clusters may change substantially for ion clusters containing more than 6 water molecules.

Based on the thermodynamic properties presented in Table 2, the presence of ammonia does not have a substantial impact on the affinity of sulfuric acid to the small clusters, the key parameter controlling atmospheric nucleation. Nevertheless, the presence of ammonia in small positive ions and the high concentrations of $\left(\mathrm{NH}_{4}\right)^{+}\left(\mathrm{H}_{2} \mathrm{O}\right)_{n}$ may have important implications for atmospheric nucleation because neutral clusters resulting from the recombination of positive and negative cluster ions, an important nucleation channel (Yu and Turco, 2008), may be ternary rather than binary and, thus, more stable. To properly assess the effect of ammonia on ionmediated nucleation, further thermochemical data for the formation of $\left(\mathrm{NH}_{4}\right)^{+}\left(\mathrm{NH}_{3}\right)_{a}\left(\mathrm{H}_{2} \mathrm{SO}_{4}\right)_{s}\left(\mathrm{H}_{2} \mathrm{O}\right)_{n}$ with $a>0, s>1$, and $n>6$ are needed.

\section{Summary}

In this paper, the effect of ammonia on the formation of positively charged ions composed of atmospheric nucleation precursors has been studied. The present study leads us to the following conclusions:

a) The protonation of ammonia has a profound effect on the stability of hydrates containing the sulfuric acid. The hydration free energies for clusters containing protonated ammonia are $\sim 2-8 \mathrm{kcal} \mathrm{mole}^{-1}$ more negative than those for $\left(\mathrm{NH}_{3}\right)\left(\mathrm{H}_{2} \mathrm{SO}_{4}\right)\left(\mathrm{H}_{2} \mathrm{O}\right)_{n}(n<6)$. Sulfu- ric acid-water binary hydrate complexes formed over $\left(\mathrm{H}_{3} \mathrm{O}^{+}\right)$are bonded stronger than those formed over $\left(\mathrm{NH}_{4}^{+}\right)$.

b) The presence of ammonia does not have a substantial impact on the affinity of sulfuric acid to the small positive ion clusters.

c) The conversions of positively charged ions $\left(\mathrm{H}_{3} \mathrm{O}\right)^{+}\left(\mathrm{H}_{2} \mathrm{O}\right)_{n-1}$ and $\left(\mathrm{H}_{3} \mathrm{O}\right)^{+}\left(\mathrm{H}_{2} \mathrm{SO}_{4}\right)\left(\mathrm{H}_{2} \mathrm{O}\right)_{n}(n<6)$ into $\left(\mathrm{NH}_{4}\right)^{+}\left(\mathrm{H}_{2} \mathrm{O}\right)_{n}$ and $\left(\mathrm{NH}_{4}\right)^{+}\left(\mathrm{H}_{2} \mathrm{SO}_{4}\right)\left(\mathrm{H}_{2} \mathrm{O}\right)_{n}$, respectively, by the addition of ammonia are favorable thermodynamically.

d) Under typical conditions in continental boundary layer, most of neutral hydrated sulfuric acid clusters do not contain ammonia and none of negative charged sulfuric acid monomer hydrates contain ammonia. In contrast, a large fraction of atmospherically relevant positive sulfuric acid monomers is expected to contain ammonia. In term of absolute concentrations, around $1000 \mathrm{~cm}^{-3}$ out of $10^{7} \mathrm{~cm}^{-3}$ of sulfuric acid momoners contain ammonia. $\left(\mathrm{NH}_{4}\right)^{+}\left(\mathrm{H}_{2} \mathrm{O}\right)_{n}$ clusters have concentrations comparable to those of $\left(\mathrm{NH}_{3}\right)\left(\mathrm{H}_{2} \mathrm{SO}_{4}\right)\left(\mathrm{H}_{2} \mathrm{O}\right)_{n}$ and appear to dominate the small positive ions. However, as a result of weak affinity of sulfuric acid molecules to small positive ions, the concentrations of both $\left(\mathrm{NH}_{4}\right)^{+}\left(\mathrm{H}_{2} \mathrm{SO}_{4}\right)\left(\mathrm{H}_{2} \mathrm{O}\right)_{n}$ and $\left(\mathrm{H}_{3} \mathrm{O}\right)^{+}\left(\mathrm{H}_{2} \mathrm{SO}_{4}\right)\left(\mathrm{H}_{2} \mathrm{O}\right)_{n}$ clusters $(n \leq 6)$ are negligible.

Further research is needed to calculate the affinity of sulfuric acid to positive ions containing $>6$ water molecules, the effect of small positive ion compositions on sulfuric acid affinity, and to assess the possible effect of ammonia on ionmediated nucleation.

Acknowledgements. The research was supported by the NSF under grant 0618124 and NOAA/DOC under grant NA05OAR4310103.

Edited by: B. Kärcher

\section{References}

Ball, S. M., Hanson, D. R., Eisele, F. L., and McMurry, P. H.: Laboratory studies of particle nucleation: Initial results for $\mathrm{H}_{2} \mathrm{SO}_{4}$, $\mathrm{H}_{2} \mathrm{O}$, and $\mathrm{NH}_{3}$ vapors, J. Geophys. Res., 104, 23709-23718, 1999.

Christensen, P. S., Wedel, S., and Livbjerg, H.: The kinetics of the photolytic production of aerosols from $\mathrm{SO}_{2}$ and $\mathrm{NH}_{3}$ in humid air, Chem. Eng. Sci., 49, 4605-4614, 1994.

Ding, C.-G., Laasonen, K., and Laaksonen, A.: Two sulfuric acids in small water clusters, J. Phys. Chem. A., 107(41), 8648-8662, 2003.

Froyd, K. D. and Lovejoy, E. R.: Experimental thermodynamics of cluster ions composed of $\mathrm{H}_{2} \mathrm{SO}_{4}$ and $\mathrm{H}_{2} \mathrm{O}$. 1. Positive ions, J. Phys. Chem. A, 107, 9800-9811, 2003.

Hirsikko, A., Bergman, T., Laakso, L., Dal Maso, M., Riipinen, I., Hñrrak, U., and Kulmala, M.: Identification and classification 
of the formation of intermediate ions measured in boreal forest, Atmos. Chem. Phys., 7, 201-210, 2007,

http://www.atmos-chem-phys.net/7/201/2007/.

Jiang, J. C.: Ab initio studies of $\mathrm{NH}_{4}^{+}\left(\mathrm{H}_{2} \mathrm{O}\right)_{1-5}$ and the influence of hydrogen-bonding nonadditivity on geometries and vibrations, $\mathrm{J}$. Phys. Chem. A, 103, 3123-3136, 1999.

Kim, T. O., Ishida, T., Adachi, M., Okuyama, K., and Seinfeld, J. H.: Nanometer-Sized Particle Formation from $\mathrm{NH}_{3} / \mathrm{SO}_{2} / \mathrm{H}_{2} \mathrm{O} /$ Air Mixtures by Ionizing Irradiation, Aerosol Sci. Tech., 29, 112-125, 1998.

Kulmala, M., Vehkamaki, H., Petaja, T., Dal Maso, M., Lauri, A., Kerminen, V.-M., Birmili, W., and McMurry, P. H.: Formation and growth rates of ultrafine atmospheric particles: A review of observations, J. Aerosol Sci., 35, 143-176, 2004.

Kurten, T., Sundberg, M. R., Vehkamäki, H., Noppel, M., Blomqvist, J., and Kulmala, M.: Ab initio and density functional theory reinvestigation of gas-phase sulfuric acid monohydrate and ammonium hydrogen sulfate, J. Phys. Chem. A, 110, 7178-7188, 2006.

Kurtén, T., Torpo, L., Sundberg, M. R., Kerminen, V.-M., Vehkamäki, H., and Kulmala, M.: Estimating the $\mathrm{NH}_{3}: \mathrm{H}_{2} \mathrm{SO}_{4}$ ratio of nucleating clusters in atmospheric conditions using quantum chemical methods, Atmos. Chem. Phys., 7, 2765-2773, 2007a,

http://www.atmos-chem-phys.net/7/2765/2007/.

Kurten, T., Torpo, L., Ding, C.-G., Vehkamäki, H., Sundberg, M. R., Laasonen, K., and Kulmala, M.: A density functional study on water-sulfuric acid-ammonia clusters and implications for atmospheric cluster formation, J. Geophys. Res., 112(D4), D04210, doi:10.1029/2006JD007391, 2007b.

Kurten, T., Noppel, M., Vehkamäki, H., Salonen, M., and Kulmala, M.: Quantum chemical studies of hydrate formation of $\mathrm{H}_{2} \mathrm{SO}_{4}$ and $\mathrm{HSO}_{4}^{-}$, Boreal Environ. Res., 12, 431-453, $2007 \mathrm{c}$.

Lewandowski, H., Koglin, E., and Meier, R. J.: Computational study of the infrared spectrum of acetic acid, its cyclic dimer, and its methyl ester, Vib. Spectrosc., 39, 15-22, 2005.

Lovejoy, E. R., Curtius, J., and Froyd, K. D.: Atmospheric ioninduced nucleation of sulfuric acid and water, J. Geophys. Res., 109, D08204, doi:10.1029/2003JD004460, 2004.

Merikanto, J., I. Napari, H. Vehkamäki, T. Anttila, M. Kulmala: New parameterization of sulfuric acid-ammonia-water ternary nucleation rates at tropospheric conditions, J. Geophys. Res. 112, D15207, doi:10.1029/2006JD007977, 2007.

Müller, A., Losada, M., and Leutwyler, S.: Ab initio benchmark study of (2-pyridone)2, a strongly bound doubly hydrogenbonded dimer, J. Phys. Chem. A, 108(1), 157-165, 2004.

Nadykto, A. B., Al Natsheh, A., Yu, F., Mikkelsen, K. V., and Ruuskanen, J.: Quantum nature of the sign preference in ion-induced nucleation, Phys. Rev. Lett., 96(12), 125701, doi:10.1103/PhysRevLett.96.125701, 2006.

Nadykto, A. B. and Yu, F.: Strong hydrogen bonding between atmospheric nucleation precursors and common organics, Chem. Phys. Lett., 435, 14-18, 2007.

Nadykto, A. B., Al Natsheh, A., Yu, F., Mikkelsen, K. V., and Herb, J.: Computational quantum chemistry: A new approach to atmospheric nucleation, Adv. Quantum Chem., 55, 449-478, 2008a.

Nadykto, A. B., Yu, F., and Herb, J.: Effect of ammonia on the gasphase hydration of common atmospheric ion $\mathrm{HSO}_{4}^{-}$, Int. J. Mol. Sci., 9(11), 2184-2193, doi:10.3390/ijms9112184, 2008 b.
Nadykto, A. B., Yu, F., and Herb, J.: Theoretical analysis of the gas-phase hydration of common atmospheric pre-nucleation $\left(\mathrm{HSO}_{4}^{-}\right)\left(\mathrm{H}_{2} \mathrm{O}\right)_{n}$ and $\left(\mathrm{H}_{3} \mathrm{O}^{+}\right)\left(\mathrm{H}_{2} \mathrm{SO}_{4}\right)\left(\mathrm{H}_{2} \mathrm{O}\right)_{n}$ cluster ions, Chem. Phys., 360, 67-73, doi:10.1016/j.chemphys.2009.04.007, 2009.

Napari, I., Noppel, M., Vehkamäki, H., and Kulmala, M.: Parametrization of ternary nucleation rates for $\mathrm{H}_{2} \mathrm{SO}_{4}-\mathrm{NH}_{3}-\mathrm{H}_{2} \mathrm{O}$ vapors, J. Geophys. Res., 107, 4381, doi:10.1029/2002JD002132, 2002.

Noppel, M., Vehkamäki, H., and Kulmala, M.: An improved model for hydrate formation in sulfuric-acid water nucleation, J. Chem. Phys, 116, 218-228, 2002.

Ortega, I. K., Kurtén, T., Vehkamäki, H., and Kulmala, M.: The role of ammonia in sulfuric acid ion induced nucleation, Atmos. Chem. Phys., 8, 2859-2867, 2008,

http://www.atmos-chem-phys.net/8/2859/2008/.

Pickard IV, F. C., Dunn, M. E., and Shields, G. C.: Comparison of model chemistry and density functional theory thermochemical predictions with experiment for formation of ionic clusters of the ammonium cation complexed with water and ammonia; atmospheric implications, J. Phys. Chem. A, 109(22), 4905-4910, 2005.

Riipinen, I., Sihto, S.-L., Kulmala, M., Arnold, F., Dal Maso, M., Birmili, W., Saarnio, K., Teinilä, K., Kerminen, V.-M., Laaksonen, A., and Lehtinen, K. E. J.: Connections between atmospheric sulphuric acid and new particle formation during QUEST III-IV campaigns in Heidelberg and Hyytil, Atmos. Chem. Phys., 7, 1899-1914, 2007, http://www.atmos-chem-phys.net/7/1899/2007/.

Smith, J. N., Moore, K. F., Eisele, F. L., Voisin, D., Ghimire, A. K., Sakurai, H., and McMurry, P. H.: Chemical composition of atmospheric nanoparticles during nucleation events in Atlanta, J. Geophys. Res., 110, D22S03, doi:10.1029/2005JD005912, 2005.

Yu, F.: The effect of ammonia on new particle formation: A kinetic $\mathrm{H}_{2} \mathrm{SO}_{4}-\mathrm{H}_{2} \mathrm{O}-\mathrm{NH}_{3}$ nucleation model constrained by laboratory measurements, J. Geophys. Res., 111, D01204, doi:10.1029/2005JD005968, 2006a.

Yu, F.: From molecular clusters to nanoparticles: second-generation ion-mediated nucleation model, Atmos. Chem. Phys., 6, 51935211, 2006b, http://www.atmos-chem-phys.net/6/5193/2006/.

Yu, F.: An improved quasi-unary nucleation model for binary $\mathrm{H}_{2} \mathrm{SO}_{4}-\mathrm{H}_{2} \mathrm{O}$ homogeneous nucleation, J. Chem. Phys., 127, 054301, doi:10.1063/1.2752171, 2007.

Yu, F. and Turco, R. P.: Ultrafine aerosol formation via ionmediated nucleation, Geophys. Res. Lett., 27, 883-886, 2000.

Yu, F. and Turco, R.: Case studies of particle formation events observed in boreal forests: implications for nucleation mechanisms, Atmos. Chem. Phys., 8, 6085-6102, 2008, http://www.atmos-chem-phys.net/8/6085/2008/.

Yu, F., Wang, Z., Luo, G., and Turco, R.: Ion-mediated nucleation as an important global source of tropospheric aerosols, Atmos. Chem. Phys., 8, 2537-2554, 2008, http://www.atmos-chem-phys.net/8/2537/2008/.

Zhang, R. Y., Suh, I., Zhao, J., Zhang, D., Fortner, E. C., Tie, X. X., Molina, L. T., and Molina, M.: Atmospheric new particle formation enhanced by organic acids, Science, 304, 1487-1490, 2004. 